\title{
Metabolismo energético em atletas de endurance é diferente entre os sexos
}

\author{
Energetic metabolism in endurance athletes \\ is different between sexes
}

\author{
Alessandra Barreto PARAVIDINO ${ }^{1}$ \\ Emilson Souza PORTELLA ${ }^{1}$ \\ Eliane de Abreu SOARES ${ }^{1,2}$
}

\section{RE S U M O}

Atletas de endurance têm suas necessidades energéticas aumentadas devido ao seu alto gasto energético durante o exercício. Contudo, ainda não estão claros quais são as diferenças do metabolismo energético entre os sexos e se essas diferenças vão implicar em mudanças em suas dietas. Sendo assim, o objetivo deste trabalho foi reunir informações da literatura sobre as diferenças entre os sexos em relação ao metabolismo energético em atletas de endurance. Alguns estudos demonstram que, durante exercício de longa duração, as mulheres utilizam como fonte de energia maior quantidade de lipídeos e menor de carboidratos e proteínas, quando comparadas aos homens. Já outros autores não encontraram diferença entre os sexos na proporção dos substratos metabolizados, mas sim nos tipos de lipídeos utilizados. Essa diferença na utilização de substratos vem sendo relacionada a distintas concentrações de alguns hormônios entre homens e mulheres, como: $17 \beta$-estradiol, progesterona, testosterona, epinefrina, norepinefrina, hormônio de crescimento, insulina e glucagon. Também foi observado que os atletas aumentam seus estoques de glicogênio quando consomem dieta com sobrecarga glicídica, enquanto que as atletas apenas têm seus estoques de glicogênio aumentados quando ingerem dieta com sobrecarga glicídica e hiperenergética. Esse achado é bastante relevante, pois a concentração de glicogênio está diretamente ligada ao rendimento do atleta durante o exercício de endurance. Portanto, a literatura sugere que há diferenças entre os sexos quanto à utilização de substratos em atletas. Porém, novos estudos são necessários para melhor esclarecimento do metabolismo energético dos atletas de endurance possibilitando, assim, a adequação de suas recomendações nutricionais.

Termos de indexação: alimentos para praticantes de atividade física; atletas de endurance; metabolismo energético; sexo.

\section{A B S T R A C T}

Endurance athletes have higher energy needs because they spend a lot of energy during exercise. However, the metabolic differences between genders and if these differences will imply in dietary changes are still not clear.

\footnotetext{
1 Universidade do Estado do Rio de Janeiro, Instituto de Nutrição, Curso Nutrição. R. São Francisco Xavier, 524, 12 andar, Bloco D, Maracanã, 20550-013, Rio de Janeiro, RJ, Brasil. Correspondência para/Correspondence to: E.A. SOARES.

${ }^{2}$ Universidade do Estado do Rio de Janeiro, Instituto de Nutrição. Rio de Janeiro, RJ, Brasil.
} 
Thus, the objective of this study was to gather information from the literature on the differences between the genders regarding energy metabolism in endurance athletes. Some studies show that during long-lasting exercise, women use more lipids and less carbohydrates and proteins as energy source when compared with men. Yet, other authors have not found a difference between genders in the proportions of metabolized substrates but in the kinds of lipids used. This difference in the use of substrates has been related with distinct concentrations of some hormones in men and women, such as $17 \beta$-estradiol, progesterone, testosterone, epinephrine, norepinephrine, growth hormone, insulin and glucagon. It has also been observed that male athletes increase their glycogen stores when they consume diets with a high sugar load while females only increase their glycogen stores when they consume diets with a high sugar load and excess calories. This finding is very relevant since the concentration of glycogen is directly associated with the athlete's performance during endurance exercise. Therefore, literature suggests that there are differences between the genders regarding the use of substrates in athletes. However, new studies are needed to better clarify the energy metabolism of endurance athletes and thus make more adequate nutritional recommendations.

Indexing terms: foods for persons engaged in physical activities; endurance athletes; energy metabolism; sex.

\section{N T R O D U Ç Ã O}

Estudos recentes demonstram que mulheres oxidam proporcionalmente maior quantidade de lipídeos e menor de carboidratos ${ }^{1-10}$ e de proteínas durante exercícios de longa duração, quando comparadas aos homens. Tal fato justificaria uma possível diferença entre os sexos na recomendação de macronutrientes em esportes de longa duração. Contudo, outros autores não encontraram diferenças significativas, entre homens e mulheres, na proporção dos substratos utilizados durante o exercício ${ }^{11-19}$.

Roepstorff et al. ${ }^{12}$ destacaram que a contribuição relativa de carboidratos e lipídeos como combustíveis, durante o exercício submáximo, é resultado da utilização dos diferentes carboidratos (glicose sangüínea e glicogênio muscular) e dos lipídeos (ácidos graxos de cadeia longa ligados à albumina plasmática, ácidos graxos dos triacilgliceróis presentes nas lipoproteínas de muito baixa densidade -VLDL- e ácidos graxos dos triacilgliceróis das células musculares). Eles também afirmaram que o estudo de utilização de substrato feito no músculo ativo oferece mais detalhes do que a avaliação de todo o corpo, refletindo apenas o metabolismo do músculo esquelético.

Alguns autores afirmaram que as concentrações basais de glicogênio muscular são similares

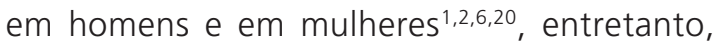
estudos sugerem diferenças entre os sexos na utilização do glicogênio hepático e não do glicogênio muscular esquelético ${ }^{2,6,12,16,21}$.
Ao contrário do glicogênio muscular, o conteúdo e a utilização de triacilglicerol intramuscular é maior nas mulheres, quando comparado aos homens ${ }^{13,22}$. Aquelas também parecem apresentar maior eficiência na lipólise e na captação de ácidos graxos livres do plasma ${ }^{12}$.

Vários fatores implicam em diferentes respostas fisiológicas ao exercício entre os sexos, como: proporção e composição corporal, características musculares, capacidade aeróbica, diferenças na atividade enzimática e mecanismos celulares, e efeito hormonal23,24.

A maior concentração de $17 \beta$-estradiol nas mulheres vem sendo considerada um dos mediadores dessa diferença entre os sexos, na preferência de substratos ${ }^{13,25-27}$. Além desse, outros hormônios vêm sendo citados como possíveis mediadores, como: progesterona, testosterona, catecolaminas (epinefrina e norepinefrina), hormônio de crescimento, insulina e glucagon 1,4,13,25,28.

O ciclo menstrual parece influenciar a utilização de carboidratos nas mulheres durante o exercício ${ }^{29}$, mas não altera o metabolismo de lipídeos ${ }^{30}$. É importante observar que a maioria dos estudos vem comparando homens e mulheres, quando estas estão na fase folicular do período menstrual (momento em que as concentrações de $17 \beta$-estradiol estão relativamente baixas) $)^{2-4,7,12,13,15,20,31-34}$. As diferenças entre os sexos podem ser maiores quando as mulheres são avaliadas na fase luteal do ciclo menstrual ${ }^{23}$. 
Carter et al. ${ }^{31}$ ressaltaram a importância do controle, não só do período do ciclo menstrual, como do fornecimento de dietas isoenergéticas, da duração e da intensidade do treinamento, quando estudos de comparação entre os sexos são realizados. O consumo de oxigênio em relação à massa magra deve ser igual para ambos os sexos.

Portanto, o objetivo deste trabalho foi reunir informações da literatura sobre as diferenças entre os sexos em relação ao metabolismo energético em atletas de endurance.

Para a realização do estudo foram consultados, dos últimos 15 anos, artigos científicos e periódicos publicados em bases bibliográficas disponíveis a partir de websites como o <http:// www.bireme.br>, que busca dados no LILACS e Medline, <http://www. periodicos.capes.gov.br>, que disponibiliza dados do Medline, FSTA, CAB Abstract, AGRIS, Web of Science e ainda o <http: //www.scielo.br>. As palavras-chave pesquisadas foram: atletas de endurance, sexos e substratos energéticos.

\section{Influência hormonal na utilização de substratos}

Os hormônios são reguladores fisiológicos que aceleram ou diminuem a velocidade de reações e funções biológicas, e essas mudanças de velocidade são fundamentais no funcionamento do corpo humano. O exercício serve de estímulo para a secreção de determinados hormônios, e de fator inibitório para outros. Entre os hormônios que têm suas concentrações aumentadas durante o exercício estão o hormônio do crescimento $(\mathrm{GH})$, o hormônio tíreo-estimulante $(\mathrm{TSH})$, a prolactina (PRL), a vasopressina $(\mathrm{ADH})$, o hormônio tireóideo, o hormônio paratireóideo (PTH), as catecolaminas, a aldosterona, o cortisol, o glucagon, a testosterona e os estrogênios ${ }^{35}$.

As catecolaminas e a insulina são os principais hormônios plasmáticos reguladores da lipólise em humanos. As catecolaminas regulam a lipólise pela estimulação de receptores $\alpha \mathrm{e}$ $\beta$-adrenérgicos podendo, portanto, diminuir ou aumentar a lipólise, dependendo de sua concentração e da afinidade de ligação do receptor. Durante o exercício, o aumento de catecolaminas circulantes estimula a lipólise pela ativação do receptor $\beta$-adrenérgico. Já a insulina é um potente inibidor da lipólise, e um pequeno aumento da sua concentração no plasma pode suprimir a taxa lipolítica a níveis muito baixos ${ }^{36}$.

Além de regular a lipólise, as catecolaminas também estimulam a glicogenólise, tanto no fígado quanto no músculo em exercício, a liberação de glicose e ácidos graxos livres para a corrente sanguínea, e a vasodilatação nos músculos em exercício ${ }^{35}$.

Durante o exercício de endurance, alguns autores encontraram maiores concentrações de catecolaminas nos homens ${ }^{1,4,13,25,28}$ enquanto que nas mulheres a insulina estava em maior quantidade $^{1,13}$. Porém, outros não encontraram diferenças entre os sexos nas concentrações desses hormônios ${ }^{3,12}$.

Horton et al. ${ }^{4}$ estudaram 28 indivíduos treinados e não treinados, sendo 14 homens e 14 mulheres, com o objetivo de comparar o substrato oxidativo e a resposta metabólica durante $2 \mathrm{~h}$ de exercício em bicicleta a $40 \%$ do consumo máximo de oxigênio $\left(\mathrm{VO}_{2 \mathrm{MAX}}\right)$ e $2 \mathrm{~h}$ após exercício. Os indivíduos também foram avaliados em um dia controle, no qual nenhum exercício foi realizado. Os hormônios analisados (epinefrina, norepinefrina, insulina, cortisol, progesterona, estradiol e testosterona) tiveram suas concentrações significantemente modificadas com o tempo, aumentando durante o exercício e diminuindo após ele. Contudo, durante o exercício, apenas as concentrações das catecolaminas e dos hormônios gonadotróficos apresentaram diferenças significantes entre os sexos. As catecolaminas tiveram maior aumento nos homens, enquanto as concentrações de estradiol foram maiores nas mulheres. Em relação à concentração de testosterona, foi observado um maior aumento em homens do que em mulheres, mas a diferença, em ambos os grupos, não foi estatisticamente significante quando 
comparada aos valores encontrados no dia controle.

Carter et al. ${ }^{31}$ verificaram se a administração de altas doses de $17 \beta$-estradiol em 8 voluntários do sexo masculino saudáveis e praticantes de atividade física, resultaria no mesmo perfil metabólico normalmente encontrado em mulheres durante exercícios de endurance. Foram formados 2 grupos de 4 indivíduos: um grupo recebeu placebo e o outro recebeu estradiol na quantidade de $2 \mathrm{mg} / \mathrm{dia}$, durante um período de 8 dias. Os resultados demonstraram que a administração de estradiol aumentou a concentração plasmática deste hormônio e diminuiu a concentração de testosterona de forma significante. Contudo, não houve diferenças no consumo de oxigênio e no coeficiente respiratório entre os dois grupos. Portanto, a administração de estradiol não teve efeito, principalmente, em relação à lipólise e à oxidação de substratos durante exercícios de endurance nesses indivíduos.

O mesmo resultado foi encontrado por Tarnopolsky et al. ${ }^{21}$, que avaliaram o efeito da administração de estradiol por 11 dias sobre o metabolismo de substratos, durante $90 \mathrm{~min}$ de exercício em bicicleta a $60 \%$ de $\mathrm{VO}_{2 \mathrm{MA} \mathrm{x}^{\prime}}$ em 11 homens jovens e saudáveis. A concentração plasmática de estradiol desses indivíduos, após a administração do hormônio, aumentou enquanto a de testosterona diminuiu. Contudo, o metabolismo de carboidratos e lipídeos não foi alterado.

Entretanto, outros autores encontraram resultados diferentes dos mencionados anteriormente. Hamadeh et al. ${ }^{27}$ avaliaram o efeito da suplementação de estrogênio ( $2 \mathrm{mg}$ de 17ß-estradiol/ dia durante 8 dias), em 12 homens ativos, na oxidação de substratos em repouso e durante exercício de endurance (90 minutos de bicicleta a $65 \%$ de $\mathrm{VO}_{2 \mathrm{MAX}}$ ). Nos dois momentos, observaram redução de $5 \%$ a $16 \%$ na oxidação de carboidratos e de $16 \%$ na oxidação de leucina, enquanto a oxidação de lipídeos aumentou de $22 \%$ a 44\%, após suplementação de estrogênio. Posteriormente, Devries et al. ${ }^{26}$ investigaram o efeito da suplementação de $2 \mathrm{mg} /$ dia de estradiol na mobilização de glicose e no uso de glicogênio muscular livre em 11 homens ativos durante 8 dias. A administração do hormônio resultou em maior utilização de lipídeos e menor de carboidratos como fonte energética durante o exercício. A concentração de glicogênio total dos indivíduos foi reduzida, porém, a utilização de glicogênio muscular não sofreu alteração.

\section{Diferenças na utilização de carboidratos}

Os carboidratos são utilizados como fonte de carbono para a síntese de componentes celulares, depósitos de energia e elementos estruturais de células e tecidos. Além disso, são as principais fontes de energia para a maioria das células do organismo, incluindo as células do músculo esquelético durante exercício, células nervosas e eritrócitos. No homem adulto, grande parte dos carboidratos é armazenada no fígado e nos músculos, sob a forma de glicogênio e, em menor parte, sob a forma de glicose sangüínea. A glicose pode ser rapidamente mobilizada a partir do glicogênio durante o exercício ${ }^{37,38}$.

Tarnopolsky et al. ${ }^{2}$ observaram as concentrações de glicogênio muscular de 15 atletas, 7 homens e 8 mulheres, após 4 dias de dieta com $75 \%$ do valor energético total (VET) de carboidratos. Os homens tiveram um aumento de $41 \%$ nas concentrações de glicogênio muscular em resposta à dieta e, também, obtiveram melhora no desempenho $(45 \%)$ durante exercício em bicicleta a $85 \%$ de $\mathrm{VO}_{2 \mathrm{MAX}}$. Já as mulheres não aumentaram as concentrações de glicogênio e mantiveram os tempos de performance durante o exercício. Os autores apontaram a diferença na quantidade absoluta de carboidratos ingeridos como um possível fator na diferença encontrada entre os sexos. Enquanto os homens ingeriram $614 \mathrm{~g}$ do substrato, as mulheres ingeriram $370 \mathrm{~g}$, embora ambas as quantidades correspondessem a $75 \%$ do VET.

Posteriormente, Tarnopolsky et al. ${ }^{20}$ estudaram atletas de endurance ( 7 mulheres e 6 homens) para avaliar a concentração de glicogênio muscular, quando dietas com sobrecarga glicídica ( $75 \%$ do VET sob a forma de carboidra- 
tos), com sobrecarga glicídica e energética (75\% de carboidratos e aumento de $34 \%$ de energia) e dieta similar à habitual eram oferecidas. Os atletas consumiram a dieta nos quatro dias anteriores ao exercício de bicicleta, praticado durante 60 minutos a $65 \%$ de $\mathrm{VO}_{2 \mathrm{MAX}}$. As mulheres não aumentaram os estoques de glicogênio muscular em resposta à maior ingestão de carboidratos em relação à dieta habitual, mas aumentaram quando ingeriram a dieta com sobrecarga glicídica e energética, ratificando a hipótese do estudo acima. Já os homens obtiveram maiores concentrações de glicogênio muscular, tanto com a dieta de sobrecarga glicídica quanto com a de sobrecarga glicídica e energética.

Em outro estudo, Tarnopolsky et al. ${ }^{3}$ compararam a ressíntese de glicogênio muscular em atletas de ambos os sexos (8 mulheres na fase folicular do ciclo menstrual e 8 homens) após exercício de endurance ( 90 min a $65 \%$ de $\mathrm{VO}_{2 \text { MAx }}$ ). Foram oferecidos dois tipos de suplementos isoenergéticos (um suplemento contendo apenas carboidratos e o outro contendo todos os macronutrientes energéticos) e um placebo, imediatamente e 1 h após o término do exercício. Cada atleta foi submetido a 3 testes com intervalos de 3 semanas entre eles, e, em cada teste, eram oferecidos um dos suplementos ou o placebo. Os autores, comparando as duas biópsias do vastus lateralis, realizadas após o término do exercício e 4 h depois de ingerido o primeiro suplemento ou placebo, observaram que, quando os dois tipos de suplementos foram oferecidos, ambos aumentaram a ressíntese de glicogênio de forma mais rápida do que com o uso do placebo. Contudo, não houve diferenças significantes entre os sexos.

Observou-se que, quando a dieta com sobrecarga glicídica era oferecida durante 4 dias antes do exercício, diferença na síntese de glicogênio entre os sexos ocorria, porém, quando era oferecida após o exercício, ambos responderam de forma semelhante na ressíntese de glicogênio ${ }^{2,3,20}$. Contudo, vale lembrar, que neste último estudo $^{3}$, a quantidade absoluta de carboidratos oferecida para os homens era maior do que a oferecida para as mulheres.
Em um estudo realizado somente com mulheres atletas, Walker et al. ${ }^{39}$ avaliaram a capacidade em aumentar o conteúdo de glicogênio muscular e o rendimento no exercício de endurance, em resposta a uma dieta com sobrecarga glicídica ( 78\% do VET), comparada a uma dieta com quantidade moderada de carboidratos ( $48 \%$ do VET). Foram avaliadas 6 atletas durante a fase luteal do ciclo menstrual, e cada dieta foi administrada durante 7 dias antecedentes ao dia da avaliação, em bicicleta ( $80 \%$ de $V \mathrm{O}_{2 \mathrm{MAX}}$ ) até exaustão. As atletas apresentaram aumento significante do conteúdo de glicogênio muscular antes do exercício, quando a dieta de sobrecarga glicídica foi oferecida, resultando em aumento da oxidação de carboidratos durante o exercício, assim como do tempo de exaustão.

Em outro estudo, que também avaliou somente mulheres atletas, Andrews et al. ${ }^{40}$ verificaram o efeito da ingestão de carboidratos em exercícios de endurance (corrida de $24 \mathrm{~km}$ ). Oito mulheres durante a fase folicular do ciclo menstrual foram avaliadas em três momentos: utilizando placebo, suplemento de carboidratos (solução de eletrólitos com $6 \%$ do substrato) e dieta de sobrecarga glicídica (75\% do VET) com suplemento de carboidratos. No período de utilização do placebo ou do suplemento de carboidratos, a dieta consistia de $50 \%$ de carboidratos em relação ao VET. Observou-se que quando a disponibilidade do substrato foi aumentada, tanto pela dieta de sobrecarga glicídica como pela suplementação de carboidratos, as atletas aumentaram a utilização desse macronutriente durante o exercício, embora não tenha sido verificada melhora significante na performance.

Riddell et al. ${ }^{33}$ observaram a oxidação de carboidratos exógenos em 7 homens e 7 mulheres durante $90 \mathrm{~min}$ de exercício em bicicleta a 60\% de $\mathrm{VO}_{2 \text { MAX }}$. Cada atleta foi submetido a 2 testes com intervalo de uma semana entre eles, sendo que, em cada teste, era oferecido um suplemento contendo carboidratos (solução com $1 \mathrm{~g} / \mathrm{kg}^{-1}$ de $\left[{ }^{13} \mathrm{C}\right]$ glucose) ou um placebo. Nos primeiros 30 min de exercício com ingestão de placebo, as mulheres oxidaram mais lipídeos em relação aos homens. Porém, nos 60 min finais, a proporção 
de carboidratos, proteínas e lipídeos metabolizados foi similar em ambos os sexos. Durante os últimos 60min de exercício com ingestão de carboidratos, observou-se maior utilização do substrato como fonte energética nas mulheres. Considerando esses resultados, a ingestão de carboidratos durante o exercício de endurance representa uma importante fonte de energia, principalmente, para as atletas.

Posteriormente, M'Kaouar et al. ${ }^{15}$ compararam a resposta metabólica de 6 atletas homens e 6 atletas mulheres (na fase folicular do ciclo menstrual) ao exercício de bicicleta, durante 120 min a $65 \%$ de $\mathrm{VO}_{2 \text { MAx }}$. Os atletas ingeriram $\left[{ }^{13} \mathrm{C}\right]$ glicose $\left(3 \mathrm{~g} / \mathrm{kg}^{-1}\right)$ diluída em água, 20min antes e durante o exercício. Considerando o gasto energético relativo, a utilização da glicose exógena como fonte energética foi similar nos homens e nas mulheres, durante o exercício de endurance. A oxidação de carboidratos, lipídeos e proteínas também não foi diferente entre os sexos.

Resultado similar foi encontrado por Wallis et al. ${ }^{41}$, que observaram a resposta metabólica, em 8 mulheres e 8 homens treinados, à ingestão de carboidratos (1,5g de glicose/min) durante 120 min a $67 \%$ de $\mathrm{VO}_{2 \mathrm{MAx}}$. Não foi encontrada diferença significante entre os sexos na utilização dos carboidratos ingeridos. A ingestão de carboidratos também reduziu a oxidação de lipídeos em ambos os sexos.

Em outro estudo, no qual foram avaliados 5 homens e 6 mulheres (na fase luteal do ciclo menstrual), durante 25 minutos a $70 \%$ e $90 \%$ de $\mathrm{VO}_{2}$, Ruby et al. ${ }^{16}$ também não encontraram diferença significante entre os sexos na oxidação total de carboidratos e lipídeos. Porém, as mulheres utilizaram relativamente mais glicose sangüínea e menos glicogênio muscular do que os homens.

\section{Diferenças na utilização de lipídeos}

O tecido adiposo é a maior reserva energética do corpo humano e tem como importante função metabólica a hidrólise de triacilglicerol e a subseqüente liberação de ácidos graxos nãoesterificados e glicerol. A maior quantidade de triacilglicerol encontra-se no tecido adiposo, porém ele também está presente no músculo esquelético e no plasma sangüíneo. Os ácidos graxos não-esterificados, provenientes do tecido adiposo e do músculo esquelético, são fontes significativas de energia durante o exercício de endurance, e, para aumentar sua disponibilidade para o músculo, é necessária a integração de eventos neurais, hormonais, circulatórios e musculares ${ }^{36}$.

Em um estudo realizado por Roepstorff et al. ${ }^{12}$ não foram observadas diferenças entre os sexos na proporção dos substratos metabolizados durante exercício de bicicleta, durante 90min a $58 \%$ do $\mathrm{VO}_{2 M A X}$. Eles avaliaram 7 atletas mulheres, durante a fase folicular do ciclo menstrual, e 7 atletas homens por meio de isótopos estáveis e biópsia muscular. Todos tiveram suas dietas controladas em relação à composição de nutrientes e ao conteúdo energético durante os 8 dias anteriores ao do experimento. Os autores observaram que, embora o coeficiente respiratório não tenha variado entre os sexos, a diferença entre eles estava na utilização de diversas fontes de lipídeos (triglicerídeos das VLDL, triglicerídeos das células musculares e triglicerídeos presentes entre as células musculares). Nas mulheres houve oxidação, significantemente, maior de ácidos graxos provenientes dos triacilgliceróis das células musculares, em relação aos homens e, de forma similar, ácidos graxos plasmáticos. Nos homens, além dos ácidos graxos das células musculares e plasmáticos, também houve oxidação de triacilgliceróis presentes entre as fibras musculares e os triacilgliceróis das VLDL.

Outra pesquisa cujo objetivo foi avaliar o metabolismo de lipídeos em homens e mulheres durante o exercício, foi realizada por Steffensen et al. ${ }^{13}$. Esses autores avaliaram o uso de triacilglicerol muscular, durante exercício em bicicleta por 90 min a $60 \%$ do $\mathrm{VO}_{2 \mathrm{MAx}}$ em 21 mulheres na fase folicular do ciclo menstrual e 21 homens, em diferentes níveis de treinamento (não-treinados, moderadamente treinados e com treinamento de endurance). Todos tiveram suas dietas controladas durante o período de 8 dias anteriores ao dia de estudo. Durante o exercício, não houve diferença 
no coeficiente respiratório entre os sexos. Contudo, antes do exercício, as mulheres apresentaram maiores concentrações de triacilglicerol no vastus lateralis e, durante o exercício, elas utilizaram mais triacilglicerol muscular quando comparadas aos homens, sendo observado um decréscimo de $25 \%$ no estoque de triacilglicerol muscular nas mulheres. Nos homens não houve diferença nas concentrações de triacilglicerol, nos dois momentos analisados, independentemente do nível de treinamento. Os autores sugeriram que, apesar de as mulheres apresentarem menor concentração de epinefrina plasmática, quando comparada à observada nos homens, ocorre diferença entre os sexos e, neste caso, as mulheres apresentariam maior sensibilidade e/ou concentração da lipase hormônio sensível em relação à epinefrina.

Posteriormente, Kiens et al. ${ }^{32}$ avaliaram de que forma a atividade física e sexo influenciam o metabolismo lipídico em relação à lipoproteína lipase muscular (mLPL) e às proteínas ligantes de lipídeos, em indivíduos realizando exercício em bicicleta a $60 \%$ de $\mathrm{VO}_{2 \mathrm{MÁ}}$, durante 90 minutos. Participaram 24 mulheres, na fase folicular do ciclo menstrual, e 22 homens de diferentes capacidades aeróbicas (não-treinados, moderadamente treinados e atletas de endurance). Foi observada maior concentração da proteína FAT/CD36 nas mulheres em relação aos homens, independentemente do nível de atividade física. Essa proteína participa de muitas funções celulares, inclusive, no transporte, utilização e estocagem de lipídeos. Essa diferença entre os sexos pode estar relacionada ao tipo de fibra que compõe o músculo esquelético, já que as mulheres possuem maior quantidade de fibra do tipo I, a qual apresenta maior concentração de FAT/CD36. Também foi observada maior concentração de proteínas ligantes de ácidos graxos da membrana plasmática $\left(\mathrm{FABP}_{\mathrm{pm}}\right)$ no músculo esquelético dos homens atletas, em comparação com as mulheres atletas sugerindo que, durante o exercício, a utilização de ácidos graxos oriundos do plasma é maior em homens treinados do que em mulheres treinadas.

Roepstorff et al. ${ }^{34}$ avaliaram 8 homens e 9 mulheres, treinados durante 90 minutos de exercício em bicicleta a $60 \%$ de $\mathrm{VO}_{2 \mathrm{MAx}}$. Esse estudo mostrou maior proporção de fibra muscular do tipo I, fibras musculares menores e maior densidade capilar nas mulheres, comparadas aos homens. Os autores associaram a maior oxidação de lipídeos nas mulheres com a morfologia muscular específica do sexo.

\section{Diferenças na utilização de proteínas}

Embora os carboidratos e os lipídeos sejam as principais fontes energéticas para contração muscular, durante o exercício de endurance, a oxidação de proteína pode fornecer mais de $8 \%$ do gasto total de energia6,11,42. Pelos métodos de excreção de uréia ${ }^{1}$ e oxidação de aminoácidos ${ }^{6,11}$, estudos têm demonstrado que as mulheres oxidam menos proteína durante o exercício, quando comparadas aos homens.

McKenzie et al. ${ }^{6}$ estudaram o efeito do treinamento de endurance por 38 dias na utilização de leucina e no metabolismo dos macronutrientes. Foram avaliados 6 homens e 6 mulheres, jovens e saudáveis, durante 90 min de exercício

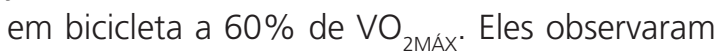
menor oxidação de leucina nas mulheres em relação aos homens. Contudo, a utilização de aminoácidos durante o exercício foi atenuada em ambos os sexos, após os 38 dias de treinamento.

Em outro estudo, Lamont et al. ${ }^{7}$ compararam o metabolismo de aminoácidos durante o exercício, em ambos os sexos, após administração de um $\beta$-bloqueador. Foram avaliados 4 homens e 4 mulheres, na fase folicular do ciclo menstrual, durante 60 min em bicicleta a $50 \%$ de $\mathrm{VO}_{2 M A ́ x}$, após 7 dias de monitoramento dietético. Foi observado que, com o uso do $\beta$-bloqueador, os homens aumentaram suas necessidades de carboidratos e aminoácidos como fonte de energia, enquanto as mulheres aumentaram a mobilização de lipídeos. Em relação ao metabolismo de aminoácidos, observou-se aumento na oxidação de leucina e na produção de lisina nos homens, e nas mulheres, não foi alterada a oxidação de leucina e houve diminuição na produção de lisina. Portanto, os autores concluíram que a preferência por substratos energéticos específicos, durante o exercício, é regulada pela atividade do receptor $\beta$-adrenérgico. 


\section{O N CLUSÃ O}

Observa-se que homens e mulheres apresentam diferenças em relação à utilização de substratos no exercício de endurance. A quantidade dos nutrientes ingeridos na dieta e a suplementação com carboidratos podem influenciar a utilização desses substratos e, possivelmente, alterar o rendimento desses atletas durante o exercício. A maioria dos estudos demonstra que as atletas oxidam, proporcionalmente, maior quantidade de lipídeos e menor de carboidratos e proteínas, quando comparadas aos atletas masculinos.

As atuais recomendações nutricionais de macronutrientes para atletas são únicas para ambos os sexos e, possivelmente, deveriam ser diferenciadas. Assim, futuros estudos são necessários para melhor conhecimento dos mecanismos responsáveis pelas diferenças entre os sexos, no que diz respeito ao metabolismo energético em atletas de endurance.

\section{REFER Ê NCIAS}

1. Tarnopolsky LJ, MacDougall JD, Atkinson AS, Tarnopolsky MA, Sutton JR. Gender differences in substrate for endurance exercise. J Appl Physiol. 1990; 68(1):302-8.

2. Tarnopolsky MA, Atkinson AS, Phillips SM, MacDougall JD. Carbohydrate loading and metabolism during exercise in men and women. J Appl Physiol. 1995; 78(4):1360-8.

3. Tarnopolsky MA, Bosman M, MacDonald JR, Vandeputte D, Martin J, Roy BD. Postexercise protein-carbohydrate and carbohydrate supplements increase muscle glycogen in men and women. J Appl Physiol. 1997; 83(6):1877-83.

4. Horton TJ, Pagliassotti MJ, Hobbs K, Hill JO. Fuel metabolism in men and women during and after long-duration exercise. J Appl Physiol. 1998; 85(5):1823-32.

5. Carter SL, Rennie C, Tarnopolsky MA. Substrate utilization during endurance exercise in men and women after endurance training. Am J Physiol Endocrinol Metab. 2001; 280(6):E898-907.

6. McKenzie S, Phillips SM, Carter SL, Lowther S, Gibala MJ, Tarnopolsky MA. Endurance exercise training attenuates leucine oxidation and BCOAD activation during exercise in humans. Am J Physiol Endocrinol Metab. 2000; 278(4):E580-7.
7. Lamont LS, McCullough AJ, Kalhan SC. Gender differences in the regulation of amino acid metabolism. J Appl Physiol. 2003; 95(3):1259-65.

8. Knechtle B, Müller G, Willmann F, Kotteck K, Eser P, Knecht $\mathrm{H}$. Fat oxidation in men and women endurance athletes in running and cycling. Int J Sports Med. 2004; 25(1):38-44.

9. Tarnopolsky MA. Gender differences in substrate metabolism during endurance exercise. Can J Appl Physiol. 2000; 25(4):312-7.

10. Venables $M C$, Achten J, Jeukendrup AE. Determinants of fat oxidation during exercise in healthy men and women: a cross-sectional study. J Appl Physiol. 2005; 98(1):160-7.

11. Phillips SM, Atkinson SA, Tarnopolsky MA, MacDougall JD. Gender differences in leucine kinetics and nitrogen balance in endurance athletes. J Appl Physiol. 1993; 75(5):2134-41.

12. Roepstorff $C$, Steffensen $C H$, Madsen $M$, Stallknecht B, Kanstrup I-L, Richter EA, et al. Gender differences in substrate utilization during submaximal exercise in endurance-trained subjects. Am J Physiol Endocrinol Metab. 2002; 282(2): E435-47.

13. Steffensen CH, Roepstorff C, Madsen M, Kiens B. Myocellular triacylglycerol breakdown in females but not in males during exercise. Am J Physiol Endocrinol Metab. 2002; 282(3):E634-42.

14. Goedecke JH, Gibson ASC, Grobler L, Collins M, Noakes TD, Lambert EV. Determinants of the variability in respiratory exchange ratio at rest and during exercise in trained athletes. Am J Physiol Endocrinol Metab. 2000; 279(6):E1325-34.

15. M'Kaouar H, Péronnet F, Massicotte D, Lavoie C. Gender difference in the metabolic response to prolonged exercise with [13C]glucose ingestion. Eur J Appl Physiol. 2004; 92(4-5):462-9.

16. Ruby BC, Coggan AR, Zderic TW. Gender differences in glucose kinetics and substrate oxidation during exercise near the lactate threshold. J Appl Physiol. 2002; 92(3):1125-32.

17. Perreault L, Lavely JM, Kittelson JM, Horton TJ. Gender differences in lipoprotein lipase activity after acute exercise. Obes Res. 2004; 12(2):241-9.

18. Perreault L, Lavely JM, Bergman BC, Horton TJ. Gender differences in insulin action after a single bout of exercise. J Appl Physiol. 2004; 97(3): 1013-21.

19. Romijn JA, Coyle EF, Sidossis LS, Rosenblatt J, Wolfe RR. Substrate metabolism during different exercise intensities in endurance-trained women. J Appl Physiol. 2000; 88(5):1707-14.

20. Tarnopolsky MA, Zawada C, Richmand LB, Carter $\mathrm{S}$, Shearer J, Graham T, et al. Gender differences in 
carbohydrate loading are related to energy intake. J Appl Physiol. 2001; 91(1):225-30.

21. Tarnopolsky MA, Roy BD, MacDonald JP, Mckenzie $S$, Martin J, Ettinger S. Short-term 17- $\beta$-estradiol administration does not affect metabolism in young males. Int J Sports Med. 2001; 22(3): 175-80.

22. Roepstorff C, Donsmark M, Thiele M, Vistisen B, Stewart G, Vissing $K$, et al. Sex differences in hormone-sensitive lipase expression, activity, and phosphorylation in skeletal muscle at rest and during exercise. Am J Physiol Endocrinol Metab. 2006; 291(5):E1106-14.

23. Marliss EB, Kreisman SH, Manzon A, Halter JB, Vranic M, Nessim SJ. Gender differences in glucoregulatory responses to intense exercise. J Appl Physiol. 2000; 88(2):457-66.

24. Mittendorfer B, Horowitz JF, Klein S. Effect of gender on lipid kinetics during endurance exercise of moderate intensity in untrained subjects. Am J Physiol Endocrinol Metab. 2002; 283(1):E58-65.

25. Tarnopolsky MA, Ruby BC. Sex differences in carbohydrate metabolism. Curr Opinion Clin Nutr Metabol Care. 2001; 4(6):521-6.

26. Devries MC, Hamadeh MJ, Grahan TE, Tarnopolsky MA. $17 \beta$-estradiol supplementation decreases glucose rate of appearance and disappearance with no effect on glycogen utilization during moderate intensity exercise in men. J Clin Endocrinol Metab. 2005; 90(11):6218-25.

27. Hamadeh MJ, Devries MC, Tarnopolsky MA. Estrogen supplementation reduces whole body leucine and cabohydrate oxidation and increases lipid oxidation in men during endurance exercise. J Clin Endocrinol Metab. 2005; 90(6):3592-9.

28. Horton TJ, Grunwald GK, Lavely J, Donahoo WT. Glucose Kinetics differ between women and men, during and after exercise. J Appl Physiol. 2006; 100(6):1883-94.

29. Devries MC, Hamadeh MJ, Phillips SM, Tarnopolsky MA. Menstrual cycle phase and sex influence muscle glycogen utilization and glucose turnover during moderate-intensity endurance exercise. Am J Physiol Regul Integr Comp Physiol. 2006; 291(4): R1120-8.

30. Horton TJ, Miller EK, Bourret K. No effect of menstrual cycle phase on glycerol or palmitate kinetics during 90 min of moderate exercise. J Appl Physiol. 2006; 100(3):917-25.

31. Carter S, McKenzie S, Mourtzakis M, Mahoney DJ, Tarnopolsky MA. Short-term 17beta-estradiol decreases glucose Ra but not whole body metabolism during endurance exercise. J Appl Physiol. 2001; 90(1):139-46.

32. Kiens B, Roepstorff C, Glatz JFC, Bonen A, Schjerling P, Knudsen J, et al. Lipid-binding proteins and lipoprotein lipase activity in human skeletal muscle: influence of physical activity and gender. J Appl Physiol. 2004; 97(4):1209-18.

33. Riddell MC, Partington SL, Stupka N, Armstrong D, Rennie C, Tarnopolsky MA. Substrate utilization during exercise performed with and without glucose ingestion in female and male endurance trained athletes. Int I Sport Nutr Exerc Metab. 2003; 13(4):407-21.

34. Roepstorff $C$, Thiele $M$, Hillig T, Pilegaard $H$, Richter EA, Wojtaszewski JF, et al. Higher skeletal muscle $\alpha_{2}$ AMPK activation and lower energy charge and fat oxidation in men than in women during submaximal exercise. J Physiol. 2006; 574(1): 125-38.

35. Canali ES, Kruel LFM. Respostas hormonais ao exercício. Rev Paul Educ Fís. 2001; 15(2):141-53.

36. Horowitz JF, Klein S. Lipid metabolism during endurance exercise. Am J Clin Nutr. 2000; 72(2):558S-63S.

37. Ribeiro BG, Pierucci APTR, Soares EA, Carmo MGT. A influência dos carboidratos no desempenho físico. Rev Bras Med Esporte. 1998; 4(6):197-202.

38. Ferreira AMD, Ribeiro BG, Soares EA. Consumo de carboidratos e lipídeos no desempenho em exercícios de ultra-resistência. Rev Bras Med Esporte. 2001; 7(2):67-74.

39. Walker JL, Heigenhauser GJF, Hultman E, Spriet LL. Dietary carbohydrate, muscle glycogen content, and endurance performance in well-trained women. J Appl Physiol. 2000; 88(6):2151-8.

40. Andrews JL, Sedlock DA, Flynn MG, Navalta JW, Ji $\mathrm{H}$. Carbohydrate loading and supplementation in endurance-trained women runners. J Appl Physiol. 2003; 95(2):584-90.

41. Wallis GA, Dawson R, Achten J, Webber J, Jeukendrup AE. Metabolic responses to carbohydrate ingestion during exercise in males and females. Am J Physiol Endocrinol Metab. 2005; 290(4):E708-15.

42. Tarnopolsky MA. Females and males: should nutritional recommendations be gender specific? Sportmedizin und Sporttraumatologie. 2003; 51(1):39-46.

Recebido em: 14/6/2005

Versão final reapresentada em: 7/12/2006 Aprovado em: 26/2/2007 
